\title{
RESEARCH OF IMAGE MATCHING ALGORITHM BASED ON ROTATION VECTOR FIELD
}

\author{
Duan YanSong \\ School of Remote Sensing and Information Engineering, Wuhan University, 129 Luoyu Road, Wuhan 430079, China
}

\author{
Commission IV, WG IV/3
}

KEY WORDS: rotation vector field, rotational invariance, image matching, time efficiency

\begin{abstract}
:
A rotation invariant image matching algorithm is introduced in this paper. The feature descriptor of feature points is calculated in polar coordinate system which achieves rotation invariant. The Wallis filter and image edge extraction are applied to reduce the influence of noise and light difference. After constructing the image feature vectors, the potential correspondences are found firstly and then the Generalized Hough Transform (GHT) is used to purify the matching result. The experiment results of three data sets show that the method is robust to image rotation, time and space efficient and is sufficient to produce matching points which can be used as initial values for further accurate image matching.
\end{abstract}

\section{INTRODUCTION}

The air vortex, side wind, nonuniform wind speed and other factors may lead to the consequence of a large rotation angle in images of aerial photography and they are inevitable in many cases. The subsequent remedy flights caused by these aerial photographs that do not meet the specifications and requirements would result in time delays and economic losses. Thus the solution of automatically large rotation angle aerial image matching and related problems has a very important significance. Matching methods based on gray correlation in conventional image matching of aerial photography have been widely used. Pyramid image strategy together with gray correlation can meet demands even if the image has small angle rotation. However, when the angle of rotation between images surpass $15^{\circ}$, this matching strategy is difficult to get the desired results. Considering the large angles of rotation in image matching, SIFT (Scale Invariant Feature Transform) operator introduced by David G. Low is the most influential algorithm currently. Compared with the traditional method based on gray scale, SIFT operator has a good rotation invariance and scale invariance. However SIFT feature matching is time-consuming and the matching accuracy is not that high. The main reason is that SIFT feature points have large amount of attributes. The time cost would be huge when traversing the characteristics of each point. In addition, SIFT operator use the minimum Euclidean distance as the similarity measurement, the overall rate of correct match is too difficult to improve. Aiming at the shortcomings of SIFT algorithm, a variety of methods have been proposed to improve it, such as: SURF (Speeded Up Robust Features), PCA-SIFT, GLOH (Gradient locationorientation histogram). These methods did much improvement on the SIFT algorithm, but the time cost and complexity of the processing are still very large.

We re-examine the aerial photography, aviation aircraft has greater immunity for rolling and pitching, so the rolling angle and pitching angle are generally not significant. The notable change exists in the yaw angle. Yaw angle results in the rotation of image plane in the image matching. As long as a feature descriptor which is not related to rotation (i.e. rotation invariant) can be found, the large rotation angle problems in the aerial image matching would be solved.

\section{THE MATCHING ALGORITHM BASED ON ROTATION VECTOR FIELD}

To establish the rotation invariant features, we create an image window which takes the target position as the center of the coordinate axis, and then a polar coordinate system is established, as showed in Figure 1, we definite the rotation vector feature as follows:

$$
F(r)=\sum_{\theta=0}^{2 \pi} G(r, \theta)
$$

Where $r$ is the radius, $\theta$ is the angle of rotation; $G$ is the attribute of the inspecting pixels, in this paper it's the image gray value.

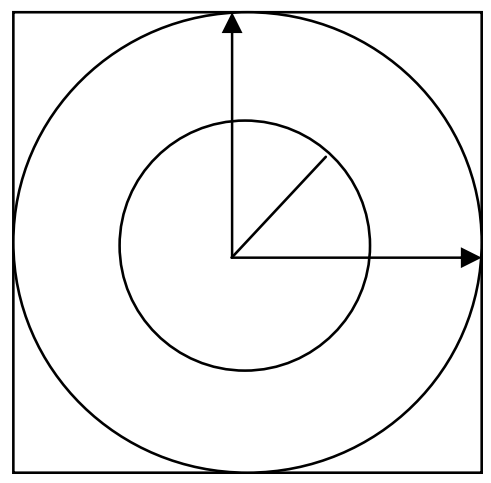

Figure 1 Rotation vector feature

When the image is rotated, pixels in the radius circle which is concentric follow this rotation. F (r) is unchanged. So, F (r) is a rotation invariant.

Based on the inspecting vector $\mathrm{F}(\mathrm{r})$, the similarity measure function is defined as follows:

$$
f=\sum_{r=0}^{R}\left|F(r)-F^{\prime}\left(r^{\prime}\right)\right|
$$

Where $F(r)$ is the reference window's rotation vector, and $F$ ' $\left(r^{\prime}\right)$ is the target window's rotation vector, when $\mathrm{f}$ equals 0 , the two features correspond. 
The chosen rotation vector $F(r)$ is described as the result of the gray value together with the radius. Actually, the target image and the reference image are not obtained under the same conditions. Subject to weather, light, the sensor and impact of other factors, the target window and the reference window usually have gray change, noise, light and contrast difference. So, for the vector $F(r)$, which has the rotation invariance, is still sensitive to these changes, as showed in Figure 2:

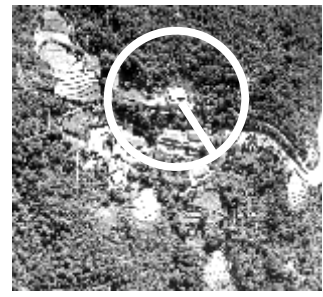

a

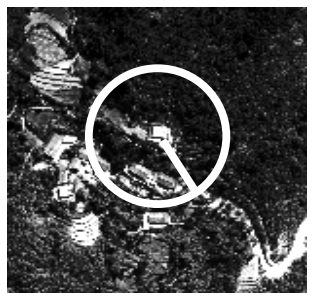

$\mathrm{b}$
Figure 2 a: $F(r)=34345, b: F(r)=12432$

As we can see, if gray-scale between left image and right image has obvious difference, $\mathrm{F}(\mathrm{r})$ is definitely different.

To reduce the gray-scale impact on the rotation vector, Wallis filter is applied. Wallis filter is used to enhance the image contrast and reduce the noise, and especially it can be used to enhance the textures in the images which are very weak in the original image (Zhang Li, Zhang 1999). Wallis filter can adjust the color difference between images. It adjusts the gray mean and variance to a given mean and variance. The formula of Wallis filtering is as follows.

$$
g(x, y)=m_{s}+v_{s}\left(g_{c}(x, y)-m_{c}\right) / v_{c}
$$

The $m_{s}$ is the aim image's mean, $v_{s}$ is aim image's variance, $\mathrm{m}_{\mathrm{c}}$ is current image's mean, $\mathrm{v}_{\mathrm{c}}$ is current image's variance, $\mathrm{g}_{\mathrm{c}}(\mathrm{x}, \mathrm{y})$ is the gray value of current image, $\mathrm{g}(\mathrm{x}, \mathrm{y})$ is the gray value after Wallis filtering operation. The mean value $\mathrm{m}$ and variance value $\mathrm{v}$ of the image is computed with formulas below.

$$
\begin{aligned}
& f_{i}(x, y)=\left\{\begin{array}{l}
0 \cdots \cdots g(x, y) !=i \\
1 \cdots \cdots g(x, y)==i
\end{array}\right. \\
& h_{i}=\sum_{x=0}^{w} \sum_{y=0}^{h} f_{i}(x, y) \\
& m=\frac{\sum_{i=0}^{k} h_{i}^{*} i}{w^{*} h} \\
& v=\sqrt{\frac{\sum_{i=0}^{k}(i-m)^{2} h_{i}}{w^{*} h}}
\end{aligned}
$$

Wallis filtering result is showed in Figure 3:

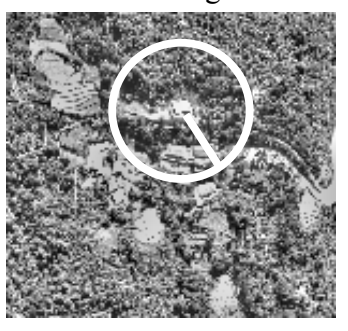

a

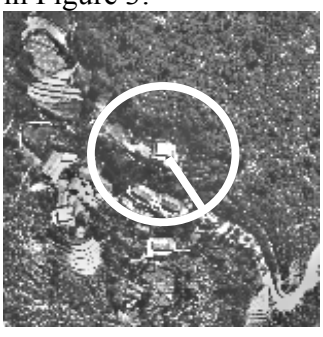

b
Figure 3 a:F(r) $=\mathbf{2 1 1 8 9 0 , ~ b : F ( r ) = 2 1 2 4 3 2 ~}$

After the filtering processing, we can obtain the new value of $\mathrm{F}(\mathrm{r})$.It can be used in the relate-computing.

In finding the correspondence in the target image, the $\mathrm{F}(\mathrm{r})$ is calculated by the same method for every image feature. F (r) can be computed for all the image features firstly, and then do the correspondence search. In this way, multi-to-multi search process can be simplified to a linear search process. As showed in Figure 4, it reflects the reference rotation vector and the target image rotation vector's distribution.

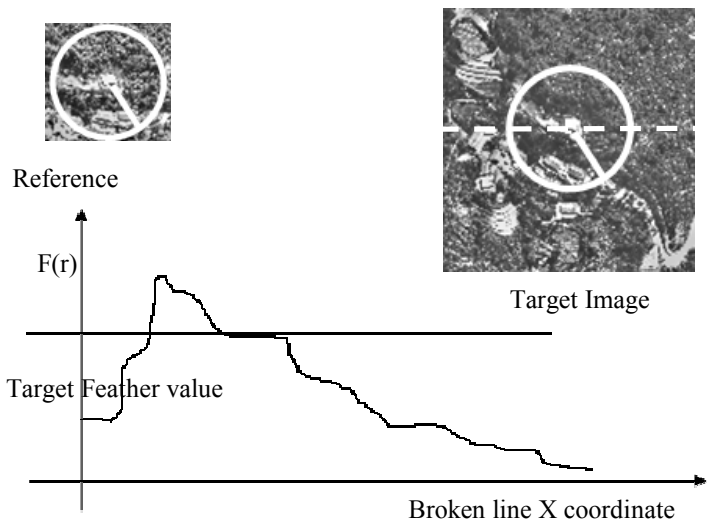

Figure 4 Target Feather and Target Image with the F(r) curve

The feature in the target image has more than one strongly related position, all these positions should be recorded as the correspondence and considered together. After completing the collection of all potential corresponding points, Generalized Hough Transform (GHT) is applied to elminate the gross errors. All points' right image coordinate $\mathrm{x}$ and $\mathrm{y}$ will subtract the left image coordinate, and the highest group of $\mathrm{dx}$ and $\mathrm{dy}$ will denote the correspondences. The points over trinal root mean square (RMS) will be removed.

\section{EXPERIMENTS AND ANALYSIS}

To verify the validity of the matching algorithm, we selected three sets of data. The detailed experimental procedure is as follows:

1) Firsty, Wallis filter is applied.

2) Then $10 * 10$ Harris feature points are extracted from the left image, each feature point contains a window with size $31 * 31$.

3) Calculates the rotation vector of these feature points, taking 15 as the radius of rotation vector.

4) Then, calculate the rotation vector of all pixels in the right image with the same method after edge extraction.

5) According to the similarity measure function, find potential feature points' correspondences. Thus, we get the same points both appearing in left and right images.

6) Analyzing the statistics of the shift which based on the Generalized Hough Transform (GHT), the peak of votes is used to establish the match point.

Following are the test data and some process results. The first image set has a $90^{\circ}$ rotation angle, the second set is $30^{\circ}$, the third one is $45^{\circ}$.

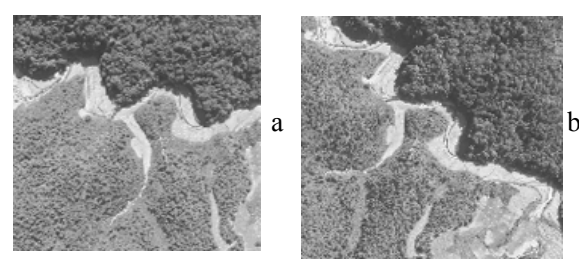

Test data 1

Area covered by trees. Size: $2048 * 2048$ 

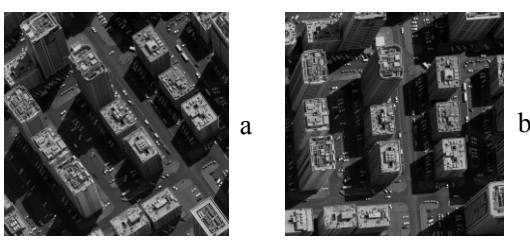

Test data 2

Area with high

buildings.

Size: $3000 * 3000$
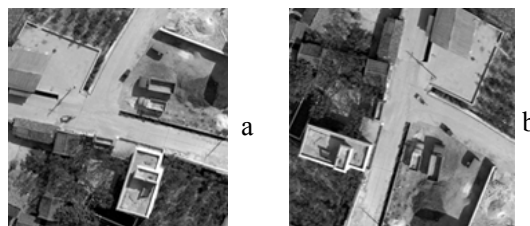

Test data 3

The normal area

Size: $4096 * 4096$

Figure 5 three test data set.

The time cost of each processing step is showed in table 1 .

Table 1 processing time

\begin{tabular}{|l|l|l|l|}
\hline \multirow{2}{*}{ Processing step } & \multicolumn{3}{|l|}{ Spending time (ms) } \\
\cline { 2 - 4 } & Data1 & Data2 & Data3 \\
\hline 1. Wallis filter & 672 & 1321 & 2321 \\
\hline 2. feature extraction (Harris) & 122 & 231 & 321 \\
\hline $\begin{array}{l}\text { 3. calculate left image vector } \\
(10 * 10 \text { points) }\end{array}$ & 32 & 35 & 37 \\
\hline $\begin{array}{l}\text { 4. calculate right image vector } \\
\text { (all pixels) }\end{array}$ & 753 & 1424 & 2531 \\
\hline 5. find potential correspondences & 12 & 12 & 11 \\
\hline 6. remove error points by GHT & 11 & 11 & 12 \\
\hline Total & 1602 & 3034 & 5233 \\
\hline
\end{tabular}

Table 2 processing time by SIFT

\begin{tabular}{|l|l|l|l|}
\hline \multirow{2}{*}{} & \multicolumn{3}{|c|}{ Spend time $(\mathrm{ms})$} \\
\cline { 2 - 4 } & Data1 & Data2 & Data3 \\
\hline Total & 42304 & 93872 & 244387 \\
\hline
\end{tabular}

(Download code form

http://www.cs.huji.ac.il/ ofirpele/SiftDist/code,

Compiling by Microsoft Visual V++6.0 )

The matching results are showed in Figure 6.

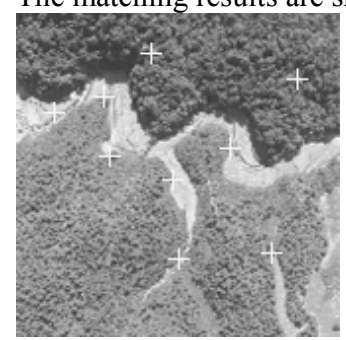

1a

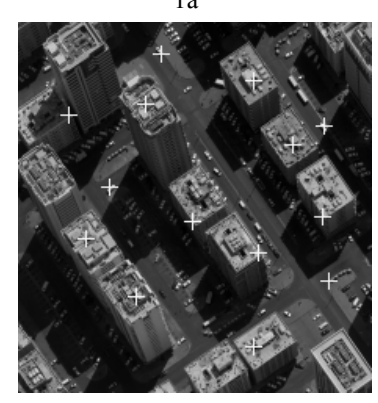

2a

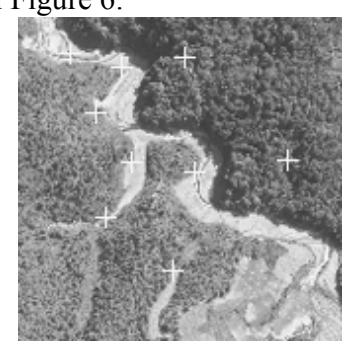

$1 \mathrm{~b}$

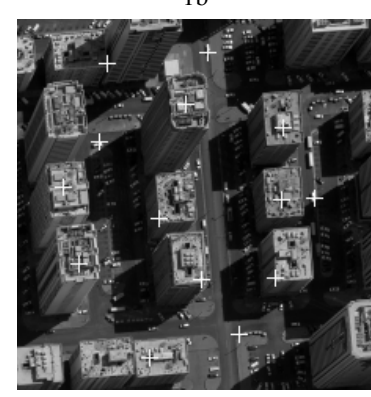

$2 \mathrm{~b}$

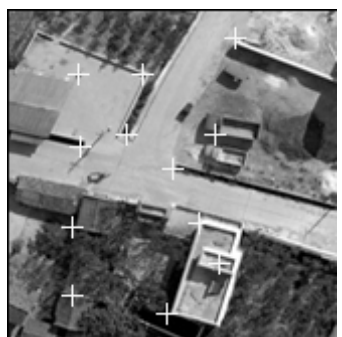

3a

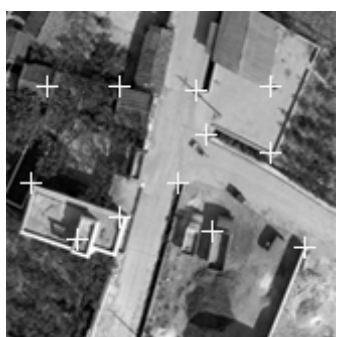

$3 b$
Figure 6 Matching Result of The Method

As can be seen from the results, the image matching algorithm based on the rotation vector field can indeed solve the problem of image rotation, and the computing speed is much faster than the traditional template matching algorithm in which each pixel is involved in complex operations ( such as convolution).

As we can see, the method can achieve good matching points which can be used lately as the initial-value for the accurate matching.

\section{CONCLUSION}

According to the above experiments, conclusions can be drawn as following.

Firstly, the matching algorithm based on rotation vector field is an optimized method which greatly reduces the computation and improves the matching speed.

Secondly, the algorithm can get good results in regions with rich features and few repeated features. And it's not suitable for regions that with poor feature or have a large number of repeated features.

Thirdly, the accuracy of the matching points obtained by this method is not that high. And these points can't directly participate in photogrammetry processing but can be used as the initial-value for the accurate matching.

Our further research will focus on the scale-change problem in the image matching which hasn't been solved yet.

\section{REFERENCES}

DAVID, G. LOWE. 2004. Distinctive Image Features from Scale-Invariant Keypoints. International Journal of Computer Vision, Vol. 60, No. 2, pp. 91-110

Herbert Bay,Tinne Tuvtellars and Luc Van Gool. 2006. SURF: Speeded Up Robust Features. European Conference on Computer Vision. pp. 404-417

Zhang Li,Zhang Zuxun,Zhang Jianqing. 1999.The Image Matching Based on Wallis Filtering. Journal of Wuhan Technical University of Surveying and Mapping, Vol, 24 No.1 ,pp. 24-26

Sim D G, Kim H K, Oh D I. 2000. Translation, scale, and rotation invariant texture descript or for texture based image retrieval .IEEE Int Conf Image Process, No.3, pp. 742- 745

Farhan Ullah, Shunichi Kaneko. 2004. Using orientation codes for rotation invariant template matching. Pattern Recognition, 37, pp. 201- 209

Yan Ke, Rahul Sukthankar. 2004. PCA2SIFT: A More Distinctive Representation for Local Image Descriptors. Proceedings of the 2004 IEEE Computer Society Conference on Computer Vision and Pattern Recognition, pp. 506-513 